\title{
Modeling of Flowable Slurry Electrodes with Combined Faradaic and Nonfaradaic Currents
}

\author{
Nathaniel C. Hoyt, Robert F. Savinell, Jesse S. Wainright
}

Department of Chemical Engineering, Case Western Reserve University, Cleveland, OH 44106, USA

\section{Abstract}

Flowable slurry electrodes have received increased interest recently for use in electrochemical energy storage and water treatment systems. In an electrochemical cell these flowing electrodes can simultaneously support both faradaic currents (through reactions occurring at the slurry/solution interface) and nonfaradaic currents (through charging of the double layer capacitance of each slurry particle as it passes through the cell). In this paper, a model based on a three-dimensional set of macrohomogeneous equations that combines the effects of both types of current is developed. The model equations are applicable to flow battery, electrochemical flow capacitor, and flow-electrode capacitive deionization systems. Using this modeling approach, the performance of slurry electrodes in symmetric electrochemical cells is examined. Scaling techniques are applied to the equations in order to permit the prediction of the steady-state current that can be achieved from a slurry electrode as a function of cell dimensions, slurry properties, flow rate, reaction kinetics, and the potential applied across the cell. Depending on the values of four dimensionless combinations of the pertinent variables (the capacitive Graetz number, the nondimensional exchange current density, the conductivity ratio, and the flow behavior index) slurry electrodes are then shown to operate in one of four distinct operational regimes. Lastly, the variation of the physical extent of the reaction zone (i.e. electroactive zone extension) with respect to the relative magnitude of advection is characterized.

\section{Introduction}

For several decades, slurry and fluidized bed electrodes have been extensively investigated for use in electrochemical systems (Heydecke \& Beck, 1990). A variety of applications have been pursued, including water treatment and electrochemical energy storage. (Appleby \& Jacquier, 1976; Baria \& Hulburt, 1973; Foller, 1986) Recent work with regard to slurry electrodes has mostly concerned three specific systems: flow batteries with flowable slurry electrodes (Duduta, et al., 2011; Fan, et al., 2014; Petek, et al. 2015), electrochemical flow 
capacitors (EFCs) (Presser, et al., 2012), and flow-electrode capacitive deionization (FCDI) systems (Jeon, et al., 2013).

Fluidized bed and slurry electrodes can support the conduction of charge through the creation of a dynamic percolation network. The current in this phase therefore acts just as it does in a porous electrode such as carbon felt or carbon paper (i.e. according Ohm’s law) (Fleischmann \& Oldfield, 1971). The flowability of the slurry, though, offers advantages that are not present for stationary porous electrodes. For example, in FCDI systems the flowability of the slurry allows for continuous operations (as the electrodes can be charged in one cell and discharged in a separate cell) and allows for simple scale-up compared to standard porous electrode CDI approaches (Jeon, et al., 2013). For hybrid flow battery systems where a plating reaction occurs on the negative electrode, the flowability of the slurry electrode allows for the energy density and power density to remain decoupled, which is not the case for porous electrodes (Petek, et al, 2015). In EFC systems, the fact that the electrode is flowing (and thereby advecting both the particles and the double layers of charge that exist at the particle/solution interface) allows for the existence of a steady-state current that can be stored for subsequent discharge (Presser, et al., 2012). The main disadvantage of

41 slurry and fluidized bed electrodes relative to porous electrodes though is their generally low electronic

42 conductivities.

43 Modeling efforts to account for the performance of electrochemical systems possessing fluidized bed and slurry 44 electrodes have also been pursued in the past. Fleischmann and Oldfield began these efforts by studying fluidized bed electrodes that supported faradaic reactions (Fleischmann \& Oldfield, 1971). In that work, standard onedimensional macrohomogeneous porous electrode equations were applied to the fluidized bed electrode and polarization relationships were derived. A three-dimensional model for a slurry supporting faradaic reactions along with coupled fluid flow and mass transfer was used by Brunini, et al. (2012) in the context of the simulation of a semi-solid flow battery. This approach consisted of including the effects of slurry rheology in the Navier-Stokes equations (which, in turn, altered the advective velocity used by the mass transport equations). Examinations of

51 rheological effects and the extension of the reaction zone due to the upstream and downstream continuation of the 52 slurry electrode away from the current collector have also been reported (Smith, et al., 2014a; Smith, et al, 2014b).

53 Combined faradaic and capacitive behavior in slurries and fluidized beds was first considered by Gabrielli, et al, 
54 (1994) who used electrochemical impedance spectroscopy to study electroactive beds. Cyclic voltammetry of stationary slurry electrodes with accompanying modeling was recently performed by Hatzell, et al. (2015).

However, advection of the surface charge (which is essential to account for the performance of EFCs and FCDI systems) was not included in any of these approaches. The work of Fleischmann and Oldfield did not do so because the fluidized bed electrode is completely contained within the cell, and so this advection is nonexistent. The work of Brunini, et al. did not do so because the flow rates they considered were very low, thereby making advection of surface charge negligible. The first attempt to model EFC performance was by Dennison, et al (2014) who included a hydraulic current in a one-dimensional equivalent circuit approach. The work of Hoyt, Wainright and Savinell (2015a) added advection of the surface charge to three-dimensional macrohomogeneous equations through the inclusion of a total derivative of the overpotential. This led to concepts of overpotential boundary layers and various associated nondimensional numbers. (Hoyt, Wainright, \& Savinell, 2015b) This previous work was directed toward the modeling of EFCs though, and, hence, it did not include Faradaic reactions.

Any model applicable to flowable slurry electrodes must include terms to account for both faradaic reactions and surface charge advection as any real slurry electrode can be expected to support both phenomena simultaneously, as has been observed experimentally. (Wu, et al., 2015) This paper will unify the previous modeling efforts to include both phenomena and will show how their simultaneous presence in a flowing slurry electrode can result in behavior distinct from what occurs when either is present alone. Numerical and analytical approaches will then be applied to the model to determine the performance characteristics of flowable slurry electrodes in electrochemical cells.

\section{Model Development}

The modeling approach applicable to a flowable slurry electrode will be developed based off of the unification of the existing modeling approaches of Brunini, et al. (2012) and Hoyt, et al. (2015b), along with nomenclature from others. (Wang, et al., 1998; Gu, et al., 2000; Devan, et al., 2004) All of these approaches are macrohomogeneous models based off of the principals of Newman, et al. (Newman \& Tobias, 1962; Newman, 1991; Newman \& Tiedemann, 1975). The combined model requires equations for fluid flow, ionic/electronic conduction, faradaic kinetics, overpotential advection, and mass transfer. After development of the general equations, a simplified set of 
nondimensional equations will be created in order to permit scaling analysis of slurry electrode performance in an

80 electrochemical cell and discernment of the governing behavior.

81

82

83

84

85

$86 \rho\left(\frac{\partial \vec{u}}{\partial t}+\vec{u} \cdot \nabla \vec{u}\right)=-\nabla p+\nabla \cdot\left(\mu_{\text {eff }}\left(\nabla \vec{u}+(\nabla \vec{u})^{T}\right)\right)$
87 Here $u$ is the shared velocity field of the respective particle and solution phases, $p$ is the pressure, $\rho$ is the mixture

88 density, and $\mu_{\text {eff }}$ is the effective viscosity of the slurry.

89

The slurries that are used as flowable electrodes typically exhibit shear-thinning behavior, and, as such, a non-

90 Newtonian rheological model must be adopted, such as the Ostwald-de Waele power-law model used by Brunini, et

91 al. (2012). In this model, the effective viscosity is described as a function of the shear rate as

$92 \quad \mu_{\text {eff }}=K \dot{\gamma}^{m-1}$

93 Here $K$ is the flow consistency index, $m$ is the flow behavior index, and $\dot{\gamma}$ is the magnitude of the rate-of-strain

94 tensor (Macosko, 1994).

95

\subsection{Electrochemical Model}

96

97

The electrochemical model for a flowable slurry electrode is based off of the macrohomogeneous approach where the slurry particles and the electrolyte are assumed to be interpenetrating homogeneous phases (Newman, 1991). For

98 the model presented below, many of the assumptions from Devan, Subramanian, and White (2004) are adopted.

99 These include the assumption that the diffusion coefficients, activity coefficients, transference numbers, double

100 layer capacitance and electrolyte volume fraction are all uniform constants. Also, the solution phase is assumed to be 
101 a binary electrolyte that can be described by concentrated solution theory. Electrolytes with more complicated

102 compositions (such as those with multiple reacting species) are not treated here, but can be modeled using

103 extensions to this approach (Johnson \& Newman, 1971).

104 A simple two-species charge transfer reaction of the canonical form (Newman, 1991) is assumed to occur at the 105 particle/solution interface:

$106 \sum_{k=1}^{2} s_{k} M_{k}^{z_{k}} \square n e^{-}$

107 where $s_{k}$ is the stoichiometric coefficient of the $k^{\text {th }}$ reactant species, $M_{k}$ is the chemical symbol for the $k^{\text {th }}$ reactant

108 species, $z_{k}$ is the charge number of the $k^{\text {th }}$ species, and $n$ is the number of electrons involved in the charge transfer 109 reaction.

110 Furthermore, as in Devan, et al. (2004), it is assumed that the total current at the slurry/solution interface can be

111 separated into individual faradaic and nonfaradaic components. Thus, charge conservation for a flowable slurry

112 electrode results in the following equations for the current density in each respective phase (with phase ' 1 '

113 indicating the percolating solid particles and phase '2' indicating the solution phase):

$114 \nabla \cdot i_{1}=-\nabla \cdot i_{2}=-a_{0} C_{d l} \frac{D\left(\Phi_{1}-\Phi_{2}\right)}{D t}-a_{0} j$

115 Here $i_{1}$ is the current density in the particle phase, $i_{2}$ is the current density in the solution phase, $a_{0}$ is the specific

116 interfacial areal density ( $\mathrm{m}^{2}$ of surface area per $\mathrm{m}^{3}$ of volume), $C_{d l}$ is the capacitance per unit interfacial area, $j$ is the

117 volumetric reaction current, $\Phi_{1}$ is the solid phase potential, and $\Phi_{2}$ is the solution phase potential. This equation for

118 slurry electrodes differs from standard macrohomogeneous porous electrode equations in that the nonfaradaic (i.e.

119 capacitive) current density is described by a total derivative instead of a partial derivative — hence accounting for 120 advection of surface charge.

121 The faradaic reaction current can be described by the Butler-Volmer equation as

$122 j=i_{0}\left[\exp \left(\frac{\alpha_{a} F}{R T} \eta\right)-\exp \left(-\frac{\alpha_{c} F}{R T} \eta\right)\right]$ 
123 Here, $i_{0}$ is the exchange current density, $\alpha_{a}$ is the anodic transfer coefficient, $\alpha_{c}$ is the cathodic transfer coefficient, $F$

124 is Faraday's constant, $R$ is the ideal gas constant, $T$ is the temperature, and $\eta$ is the overpotential, described by the

125 equation

$126 \eta=\Phi_{1}-\Phi_{2}-E_{r e f}$

127 where $E_{\text {ref }}$ is the open-circuit potential of the electrode reaction relative to a suitable reference electrode such that the 128 overpotential is zero at equilibrium.

129 In general, $i_{0}$ is dependent on the surface concentrations of the reactant species (and not on the local

130 macrohomogeneous volumetric average value of the concentration); a correlation for a mass transfer resistance can

131 be added to account for the difference between the average concentration at the surface-electrolyte interface and the

132 volumetrically-averaged concentration (Wang, Gu, \& Liaw, 1998; Trainham \& Newman, 1977), but for the sub-

133 micron to micron sized particles typically used in slurry electrodes, it is assumed that the relative magnitude of

134 diffusion is sufficient to make this effect negligible.

135 As in porous electrodes, Ohm's law is valid for the percolated solids phase of a slurry resulting in an equation for

136 the solid phase potential as (Fleischmann \& Oldfield, 1971):

$137 \quad i_{1}=-\sigma_{e f f} \nabla \Phi_{1}$

138 Here, $\sigma_{\text {eff }}$ is the effective conductivity of the slurry. Similarly, the modified Ohm's law for the solution phase results

139 in an equation for the solution phase potential as

$140 \quad i_{2}=-\kappa_{e f f} \nabla \Phi_{2}+\kappa_{D, e f f} \nabla \ln c$

141 where $c=c_{+} / v_{+}=c / v_{-}$is the electrolyte concentration, $v_{+}$is the number of moles of cations per mole of electrolyte,

$142 \kappa_{\text {eff }}$ is the effective conductivity of the ionic phase, and $\kappa_{D, \text { eff }}$ is the diffusion conductivity defined as (Gu, et al., 2000)

$143 \kappa_{D, e f f}=\frac{v R T \kappa_{e f f}}{F}\left(\frac{s_{+}}{n v_{+}}+\frac{t_{+}^{0}}{z_{+} v_{+}}\right)$ 
where $t^{o}$ is the transference number of the cation. Species conservation as applied to the solution phase results in an equation for the electrolyte concentration

$146 \varepsilon \frac{D c}{D t}=\varepsilon D_{e f f} \nabla^{2} c+\frac{a_{0}\left(1-t_{+}^{0}\right)}{z_{+} \nu_{+} F} j+\frac{a_{0} C_{d l}}{z_{+} \nu_{+} F}\left(t_{-}^{0} \frac{d q^{+}}{d q}-t_{+}^{0} \frac{d q^{-}}{d q}\right) \frac{D\left(\Phi_{1}-\Phi_{2}\right)}{D t}$

147

148

150

\section{1}

152

153

154

155

156

157

158

159

160

161

162

Depending on the relative magnitude of the system parameters, differing phenomena accounted for by these

165

Here $D_{\text {eff }}$ is the effective diffusion coefficient of the electrolyte, $\varepsilon$ is the electrolyte volume fraction, and $\left(d q^{+} / d q\right)$ and $\left(d q^{-} / d q\right)$ are related to the fraction of charge in the double layer that is a result of adsorption and desorption of either cations or anions respectively. (Johnson \& Newman, 1971) This form of the species conservation equation accounts for both consumption/generation of the reactant by the faradaic reaction and for adsorption/desorption of ions into the double layer. A more sophisticated treatment of the double layer that includes the effects of micropores is possible (as in Biesheuvel, et al. (2012) and Biesheuvel, et al. (2011)). Inclusion of this behavior into the present model would require the solution of coupled four-dimensional equations, so for now the methodology of Johnson \& Newman is assumed to be adequate.

The effective ionic conductivity and effective diffusion coefficient in Equations 9, 10, and 11 can be related to their respective bulk values, $\kappa$ and $D_{e}$, through use of Bruggeman relationships:

$\kappa_{\text {eff }}=\varepsilon^{1.5} \kappa$

$D_{e f f}=\varepsilon^{1.5} D_{e}$

Finally, the presence of a separator can be modeled as in the work of Brunini, et al. (2012) thereby completing the necessary set of equations.

\subsection{Nondimensional Model}

The above set of equations is applicable to any slurry electrode system with a binary electrolyte. Examples of applicable systems include flow batteries, EFCs, and FCDI systems.

equations may take precedence. For example, as the flow rates they considered were low, faradaic consumption of 
reactant was of major importance to the flow batteries considered by Brunini, et al. (2012), but the capacitive current mechanism arising from advection was not. Conversely, in EFC and FCDI systems, faradaic reactions are

168 unimportant.

169

170

171

172

173

174

$181 \quad j=\frac{i_{0} n F}{R T} \eta$

In the absence of concentration gradients, the modified Ohm's law for the electrolyte solution takes the simplified form $i_{2}=\kappa_{e f f} \nabla \Phi_{2}$. The combination of each per-phase charge conservation equation (Equation 5) with the respective phase's Ohmic equation (Equation 8 or 9) therefore results in the following governing equations for the electronic and ionic potentials: $\sigma_{\text {eff }} \nabla^{2} \Phi_{1}=a_{0} C_{d l} \frac{D\left(\Phi_{1}-\Phi_{2}\right)}{D t}+\frac{a_{0} i_{0} n F}{R T} \eta$

$$
\kappa_{\text {eff }} \nabla^{2} \Phi_{2}=-a_{0} C_{d l} \frac{D\left(\Phi_{1}-\Phi_{2}\right)}{D t}-\frac{a_{0} i_{0} n F}{R T} \eta
$$


Nondimensionalization of these equations is useful as doing so allows for simple determination of the relative magnitudes of the controlling phenomena. By nondimensionalizing and then combining Equations 15 and 16, a governing equation for the nondimensional overpotential, $\eta$ ', can be created:

$191 \quad \frac{D \eta^{\prime}}{D t^{\prime}}=\frac{1}{P e_{c}}\left(\nabla^{2} \eta^{\prime}-v^{2} \eta^{\prime}\right)$

192

193

194

195

$202 \quad v^{2}=\frac{a_{0} i_{0} F n}{R T}\left(\frac{1}{\kappa_{\text {eff }}}+\frac{1}{\sigma_{\text {eff }}}\right) \delta^{2}$

$P e_{c}=\frac{U \delta}{\alpha_{e}}=a_{0} C_{d l}\left(\frac{1}{\kappa_{e f f}}+\frac{1}{\sigma_{e f f}}\right) U \delta$

Here, $\eta^{\prime}=\eta / \Delta V_{0}$, where $\Delta V_{0}$ is the potential applied across the electrochemical half-cell, and $t^{\prime}=(U / \delta) t$ where $U$ is the average streamwise velocity of the slurry and $\delta$ is the gap between the current collector and the separator as shown in Figure 1 (which has also been used to nondimensionalize all of the spatial coordinates). The capacitive Péclet number, $P e_{c}$, and the nondimensional exchange current density $v^{2}$ are as defined below.

Equation 17 shows that the behavior of the nondimensional overpotential in a slurry with simultaneous faradaic and nonfaradaic capacitive currents is dictated by advection and diffusion along with a sink/source term. The relative magnitudes of each of these components is controlled by the values of the capacitive Péclet number, $P e_{c}$, and the nondimensional exchange current density $v^{2}$. These nondimensional numbers are defined as (Hoyt, Wainright, \& Savinell, 2015; Newman \& Tiedemann, 1975)

Here $\delta$ is the gap, and $\alpha_{e}=\kappa_{e f f} \sigma_{e f f} / a_{0} C_{d l}\left(\kappa_{e f f}+\sigma_{e f f}\right)$ is the electronic diffusivity (Johnson \& Newman, 1971) of the slurry. The capacitive Péclet number describes the magnitude of overpotential advection relative to overpotential diffusion. Similarly, the nondimensional exchange current density describes the magnitude of the overpotential source/sink relative to overpotential diffusion.

As later results will show, the ratio of $v^{2}$ to $P e_{c}$ (i.e. the ratio of the magnitude of the faradaic source/sink term relative to advection) is important in describing whether an electrochemical cell with slurry electrodes acts more like a flow battery or like an electrochemical flow capacitor. This term is analogous to the Damköhler number from mass 
transfer (which is the ratio of the reaction rate to the convective mass transfer rate of a reacting species), and, hence,

211 the name “capacitive Damköhler number”, $D a_{c}$, has been adopted for this quantity:

$212 D a_{c}=\frac{v^{2}}{P e_{c}}=\frac{i_{0} F n}{R T} \frac{\delta}{C_{d l} U}$

\subsection{Flow Cell Geometry and Numerical Implementation}

214 Numerical simulations and asymptotic analyses of the above governing equations allow for the prediction of the 215 combined faradaic and nonfaradaic behavior of slurry electrodes. Toward that end, slurry electrodes were modeled 216 in an electrochemical cell (Figure 1) that is representative of the existing flow cells of interest to this work. For the 217 sake of simplicity and to permit scaling analysis, the cell geometry considered is that of a two-dimensional channel.

218 This type of geometry is very similar to what is used for slurry flow batteries and electrochemical flow capacitors 219 (Presser, et al., 2012; Dennison, Gogotsi, \& Kumbur, 2014; Petek, et al., 2015; Petek, et al., 2016). Symmetric cell 220 configurations will be considered in this work as they are often used in both the study of flow batteries with slurry 221 electrodes and in the study of EFCs (Petek, et al., 2016; Hoyt, et al, 2015b), but the models are equally valid to 222 asymmetric cases. Due to this symmetry, only the positive half-cell was modeled. The applied potential across the 223 half-cell, $\Delta V_{0}$ (i.e. one-half the total potential applied across the entire cell) is considered to be constant along the 224 length of the electrode. For the sake of simplicity, the resistance from the separator was ignored as was any cell assembly or slurry-to-electrode contact resistance. Long entrance regions were included in order to ensure a fully developed velocity profile by the time the slurry reached the electrode.

The numerical approach taken is similar to that in the earlier work of Hoyt, Wainright, and Savinell (2015b). A three-dimensional solver for the coupled set of Equations 15 and 16 along with the associated non-Newtonian fluid

229 flow equations (Equations 1 and 2) was created using the OpenFOAM open source, finite volume CFD framework.

230 (OpenFOAM, 2012)

231 Numerical simulations and scaling analyses of non-Newtonian and Newtonian slurries were performed in order to 232 demonstrate the general effect that shear thinning behavior has on slurry electrode performance. 

unsteady calculation over a sufficient time period to allow the initial transients to die away.

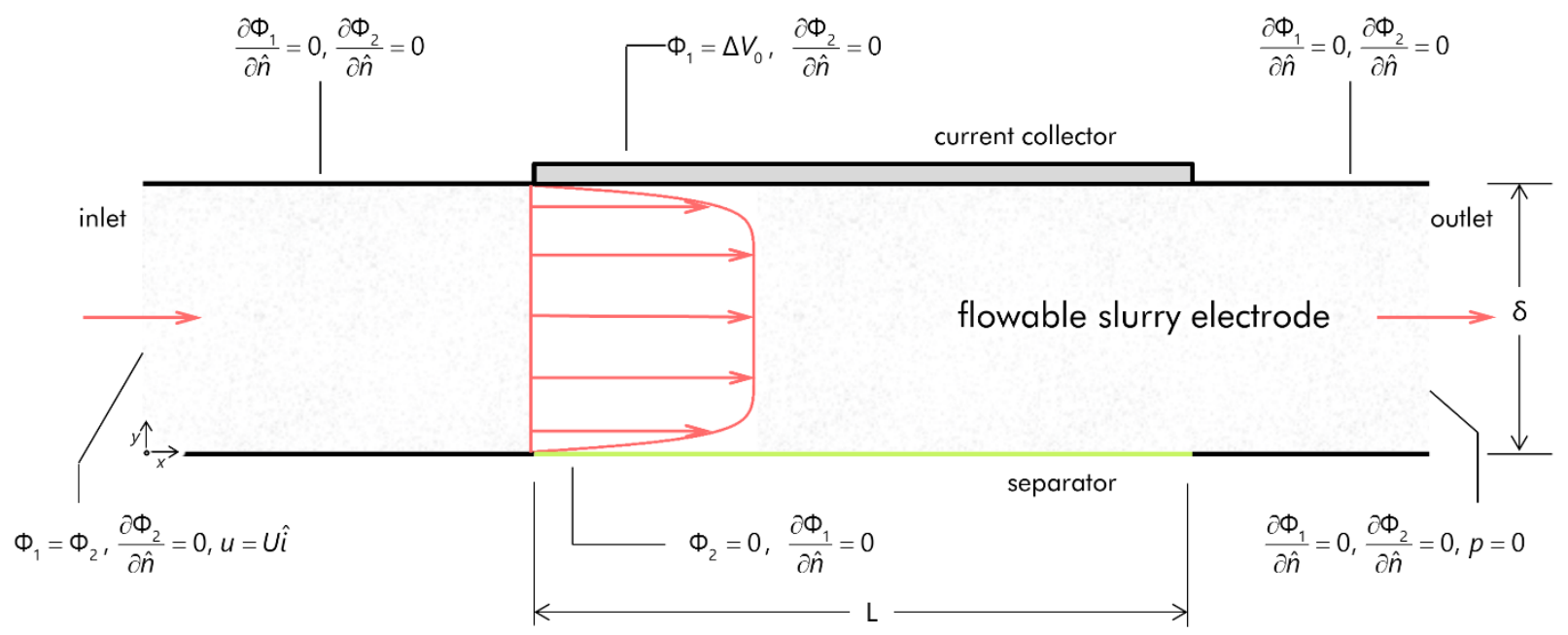

$w$ (not pictured) is the channel width (in the z-direction)

Figure 1. Setup for the positive side of the two-dimensional symmetric cell slurry flow battery. Boundary conditions are as indicated. Figure is not to scale. The fully developed velocity profile is as shown (a uniform velocity profile is used as the boundary condition at the inlet).

\section{Results and Discussion}

\subsection{Scaling Predictions}

The equations that have been developed allow for the creation of scaling predictions of the steady-state current that can be achieved from a flowing slurry electrode given specified slurry properties, cell dimensions, flow rates, reaction kinetics, and applied potential across the electrochemical cell. Prediction of this current is crucial to understanding the performance of any slurry flow battery, EFC, or FCDI system.

Similar predictions were made in the work of Hoyt, et al. (2015b) for slurries in the absence of any faradaic reactions (i.e. for the nonfaradaic capacitive current only) as applied to electrochemical flow capacitors. That work showed that the charging of an EFC shows analogous behavior to the Graetz problem from heat transfer. Specifically, the advection and diffusion of surface charge into the channel of an EFC that is generated by a potential applied across the cell is similar to the advection and diffusion of heat into a channel that is imposed by a 
temperature difference between the fluid and a heated section of wall. The total current was shown to be a function only three parameters which are defined below: the capacitive Graetz number $\left(G z_{c}\right)$, a ratio of the effective ionic and electronic conductivities $(\gamma)$, and the flow behavior index from the Ostwald-de Waele power-law $(m)$.

254

$G z_{c}=\frac{P e_{c}}{A R}=a_{0} C_{d l}\left(\frac{1}{\kappa_{e f f}}+\frac{1}{\sigma_{e f f}}\right) \frac{U \delta^{2}}{L}$

$\gamma=\left(\kappa_{e f f}+\sigma_{\text {eff }}\right)\left(1 / \kappa_{\text {eff }}+1 / \sigma_{\text {eff }}\right)$

Here $A R=L / \delta$ is the aspect ratio of the channel with electrode length $L$.

Depending on the values of $G z_{c}$ and $\gamma$, an EFC operating under charging conditions was shown to adopt the behavior of one of three possible regimes. At small $G z_{c}$, the slurry was found to act in a fully utilized mode of operation wherein the entirety of the available slurry/solution interface is fully charged to half of the total potential applied across the cell (i.e. the available area for double layer formation is charged as much as is possible). At moderate $G z_{c}$ (i.e. when $G z_{c}$ was larger than some $G z_{c, c r i t}$ ), it was found that the performance of the EFC transitioned into behavior dictated by the formation of boundary layers of overpotential that form at the current collector and separator. Finally, as $G z_{c}$ was increased further, the EFC ultimately entered an ohmically limited state where the boundary layers of overpotential become very thin and IR-losses predominate. Nondimensional asymptotic solutions to predict the steady-state current, $I$, that the EFC achieves when operating in each of these regimes were calculated, and the results were as follows:

$267 \quad I / I_{\text {diff }}=\left\{\begin{array}{lll}G z_{c} & \text { for } & G z_{c}<c_{0}^{3 / 2} \\ c_{0} G z_{c}^{1 / 3} & \text { for } & c_{0}^{3 / 2}<G z_{c}<\left(\gamma / c_{0}\right)^{3} \\ \gamma & \text { for } & G z_{c}>\left(\gamma / c_{0}\right)^{3}\end{array}\right.$

$269 \quad I_{\text {diff }}=\frac{\left(\Delta V_{0}\right)}{\left(1 / \kappa_{e f f}+1 / \sigma_{e f f}\right)}\left(\frac{L w}{\delta}\right)$ 
where $w$ is the width of the channel.

$271 c_{0}$ is a constant related to the velocity gradient at the wall and is equal to (Richardson, 1979)

$272 \quad c_{0}=\frac{[6(2+1 / m)]^{1 / 3}}{2 \Gamma(4 / 3)}$

For a Newtonian fluid (with $m=1$ ), $c_{0}$ is equal to roughly 1.467 . The shear thinning behavior associated with flowable slurry electrodes (with $m<1$ ) results in larger values for $c_{0}$ because the velocity gradient near the wall is greater in these cases for a given average streamwise velocity.

A composite solution was made from the combination of these individual equations, and by doing so the behavior of EFCs over the entire possible space of $G_{c}$ and $\gamma$ was characterized. When plotted on log-scales, this composite solution took the form of distinct line segments (as shown in Figure 3 of Hoyt, et. al. 2015b).

For a slurry that supports faradaic reactions along with the advection-induced capacitive current, a similar scaling approach can be adopted in order to discern the new behavior that arises. This new behavior includes a new regime of operation and, depending on the relative magnitude of the faradaic reactions, the elimination of old regimes associated with EFCs. Unlike the EFC situation where current was dependent only on $G z_{c}, \gamma$, and $m$, the steady-state current for a general slurry electrode possessing simultaneous faradaic reaction currents is also dependent on the nondimensional exchange current density $v^{2}$.

The relative magnitude of these faradaic reactions can be seen by examination of Equation 17 . Clearly, when $v^{2} / P e_{c}$ (i.e. $D a_{c}$ ) is very small, the slurry electrode acts in a purely capacitive manner. This is the situation that was modeled by Hoyt, et al. (2015b) for EFCs. However, for a fixed $v^{2}, P e_{c}$ (and, hence, $G z_{c}$ ) can always be made small enough such that the advective term becomes inconsequential. For this situation, the slurry electrode adopts behavior identical to a porous electrode where a Laplacian of the overpotential is exactly matched by the faradaic source/sink term. This is the situation that was modeled by Fleischmann \& Oldfield (1971) for fluidized bed electrodes (where $P e_{c}$ was zero because the solids phase was stationary).

This quasi-static, low $P e_{c}$ situation acts as an additional operational regime for a flowing slurry electrode to be added to the existing regimes seen for EFCs. Much as in the three regimes associated with an EFC, an asymptotic 


$$
\frac{I}{I_{\text {diff }}}=\gamma\left(1+\frac{2+(\gamma-2) \cosh v}{v \sinh v}\right)=I_{f}^{\prime}
$$
to be

solution to predict the current density can be found. A suitable prediction can be provided by the standard onedimensional macrohomogeneous equations used for porous electrodes. By nondimensionalizing available polarization results (Newman \& Tiedemann, 1975), the predicted asymptotic current at low $G z_{c}$ can be determined

The nondimensional current $I_{f}$ ' that results for this faradaically-dominated quasi-static regime is a function of $v^{2}$ and $\gamma$, but not of the capacitive Graetz number or the flow behavior index (as at the low $G z_{c}$ where this regime is active, the advective terms are not appreciable).

Figures 2 and 3 show the composite scaling solution that results when the prediction for the quasi-static regime is added to the existing regimes from Equation 23.

In Figure 2, $I_{f}{ }^{\prime}<c_{0}{ }^{3 / 2}$. This occurs for small values of $v^{2}$ (e.g., with relatively sluggish faradaic reactions). In this scenario, the quasistatic faradaic regime that exists at low $G z_{c}$ transitions into the fully utilized capacitive current EFC regime. Therefore, in this case the slurry electrode has four regimes.

In Figure 3, $I_{f}{ }^{\prime}>c_{0}^{3 / 2}$. This occurs for larger values of $v^{2}$ (e.g. if the charge transfer resistance of the faradaic reaction is reduced). Now, the faradaic regime extends to larger values of the capacitive Graetz number such that the fully utilized capacitive current regime is never achieved. For this fixed $v^{2}$, the slurry electrode only has three attainable regimes. Instead of transitioning from the quasi-static regime to the fully utilized regime, the slurry behavior transitions directly into the regime such that the development of the double layers of overpotential is the controlling phenomena.

In reality, the transitions between regimes will not occur at distinct points, as depicted in Figures 2 and 3, but, instead, transitional regions will exist between adjacent regimes. Comparison of the scaling results to the numerical solution of Equations 15 and 16 will show this more fully in the next section. Contour plots of the numerically predicted overpotential distribution will also be shown in the next section to demonstrate this. 
317 As stated earlier, the results in this paper are applicable to situations with negligible gradients in reactant

318 concentrations (i.e. at high stoichiometric flow rates). The above results for the predicted current can thereby

319 provide an estimate of the validity of this assumption by comparison to the maximum possible current that

320 corresponds to complete consumption of the incoming reactant. When this comparison is made, the high

321 stoichiometric flow rate criterion takes the form:

$322 \frac{I}{I_{\text {diff }}} \square \frac{n F_{C} U \delta^{2}(1 / \kappa+1 / \sigma)}{\Delta V_{0} L}$

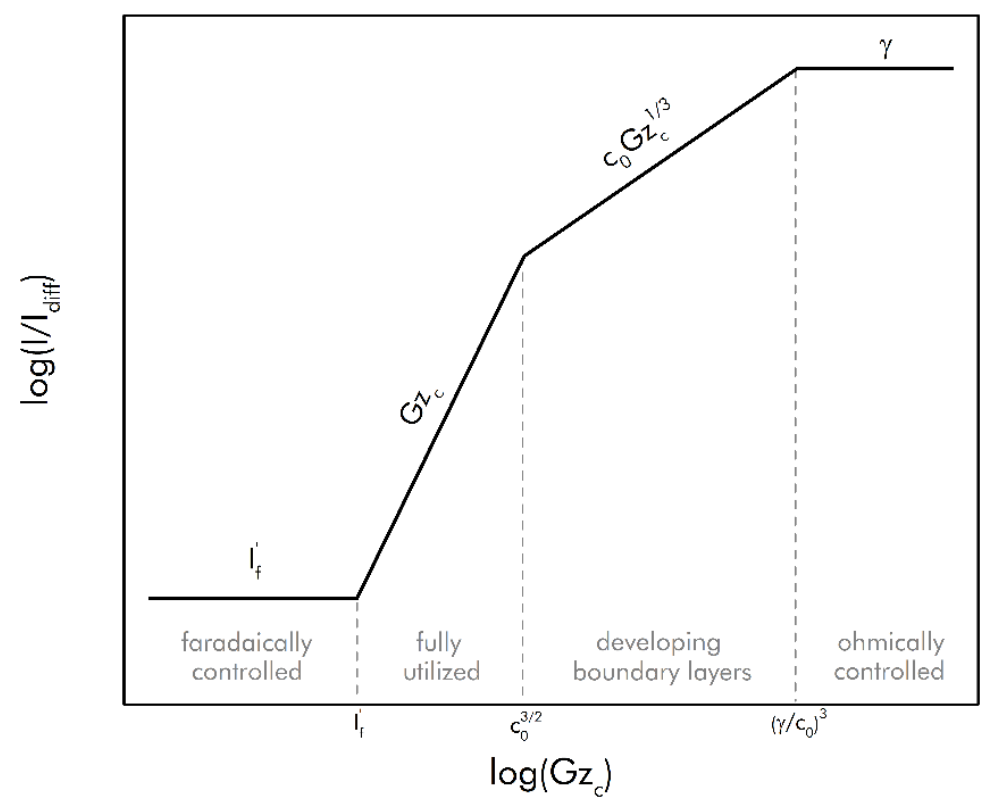

Figure 2. Prediction of the steady state current $I / I_{\text {diff }}$ using the composite construction of asymptotic scaling results from the indicated operational regimes (for $I_{f}{ }^{\prime}<c_{0}{ }^{3 / 2}$ ). All four operational regimes are present under these circumstances. 


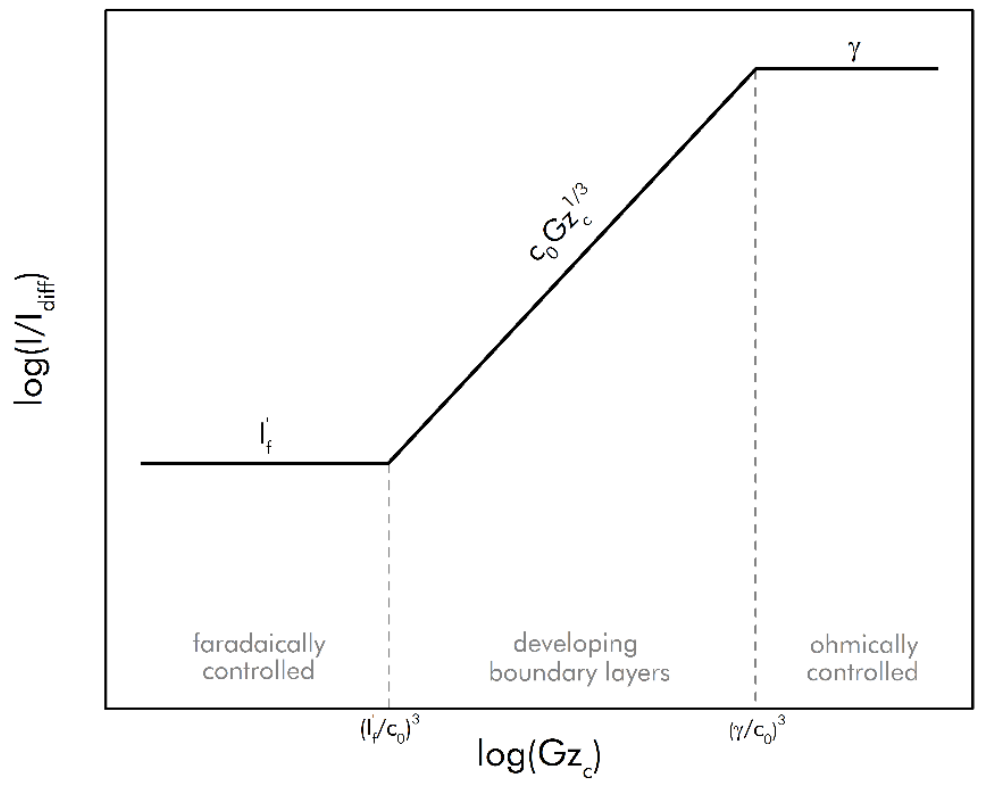

Figure 3. Prediction of the steady state current $I / I_{\text {diff }}$ using the composite construction of asymptotic scaling results from the indicated operational regimes (for $I_{f}{ }^{\prime}>c_{0}^{3 / 2}$ ). Under these circumstances, only three operational regimes are present.

\subsection{Comparison to Numerical Predictions}

In order to describe the slurry electrode operational regimes more fully and to determine the validity of the above scaling relationships, numerical simulations for the coupled set of equations (including Equations 15 and 16 and the fluid flow Equations 1 and 2) were performed for the symmetric cell flow battery geometry in Figure 1. Simulations were run for a large number of combinations of flow rate, ionic conductivity, electronic conductivity, specific capacitance, and exchange current density. The simulations were done in terms of dimensional quantities, and the results were subsequently nondimensionalized. The numerical results showed that, as expected, the predicted steadystate current was a function only of $G z_{c}, \gamma, v^{2}$, and $m$.

The numerically predicted nondimensional steady-state current versus $G z_{c}$ for a specific set of cases with $\gamma=100$ and $v^{2}=0.087$ is shown in Figure 4 along with the composite scaling solution as developed above. For these cases, $I_{f}$ ' $<c_{0}^{3 / 2}$, and, hence, all four regimes from Figure 2 exist. As can be seen, the numerical solution closely follows the asymptotic solutions, displaying the expected scaling behavior for each of the regimes before transitioning to the next. Unlike the EFC case where the scaling solution acted as an upper bound on the achievable current (Hoyt, et al., 
2015b), the scaling prediction at the transition from the quasi-static regime to the capacitive fully-utilized regime is

347 a lower bound as the faradaic and the advectively-induced capacitive currents can exist simultaneously and achieve 348 a larger combined current than either mechanism could support individually.

349 Results are shown for cases with $m=0.1$ and $m=1.0$ to show the effect that shear-thinning behavior has on the current 350 from the slurry electrode. In the quasi-static, fully utilized, and Ohmically-limited regimes, the respective curves for 351 both the Newtonian and non-Newtonian slurry overlap, as expected from the scaling predictions (because the asymptotic scaling relationships for those regimes are not dependent on $m$ ). In the developing boundary layer region though, the numerically predicted currents do not overlap, with larger currents being predicted for the shear-thinning slurry. This, again, is consistent with the scaling predictions as, for this regime, $m$ is able to alter the steady-state current through its effect on the value of $c_{0}$.

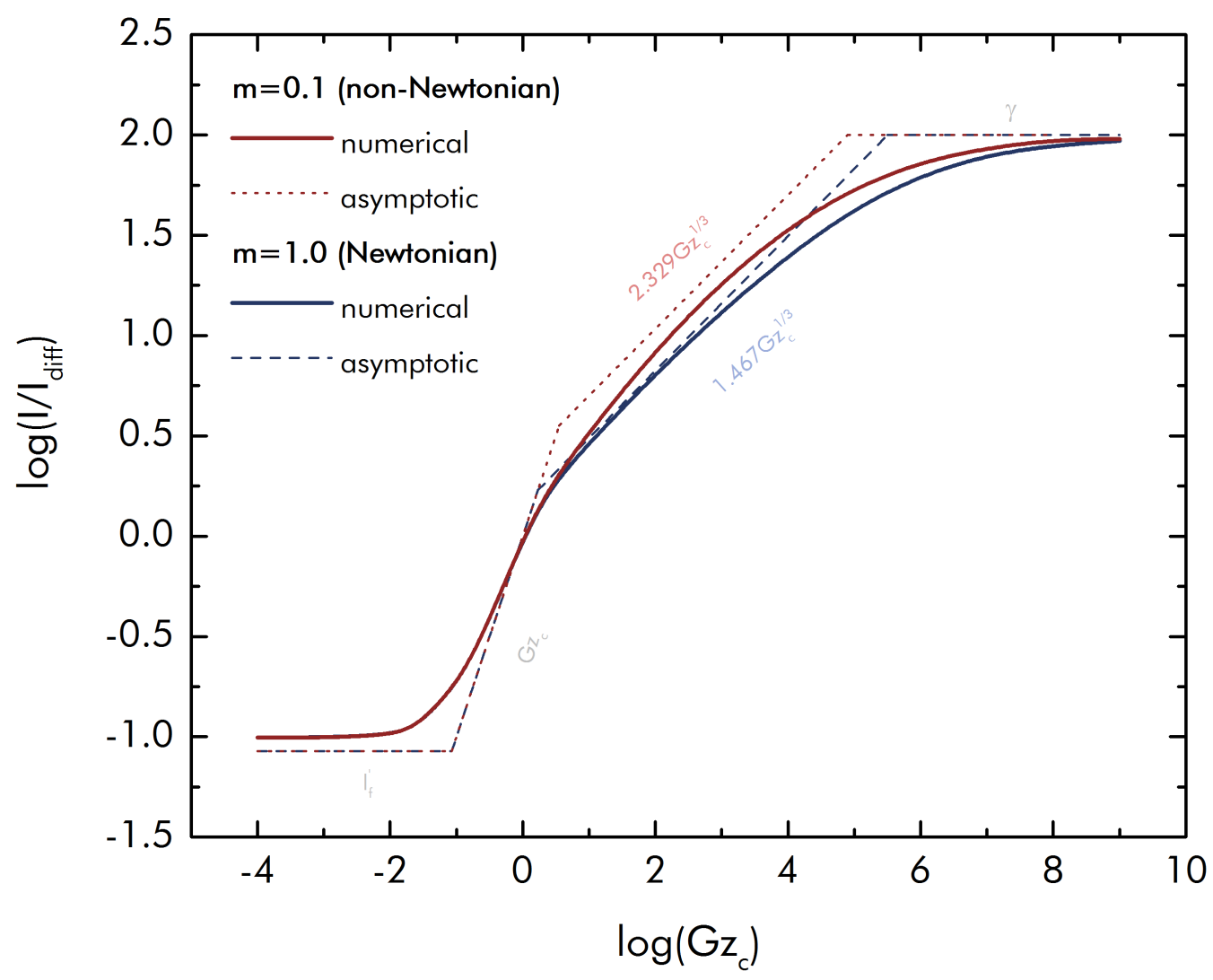

Figure 4. Nondimensional steady-state current $I / I_{\text {diff }}$ versus capacitive Graetz number $\left(G z_{c}\right)$ for $\gamma=100, v^{2}=0.087$. 
Figure 5 shows the contours of overpotential at various $G z_{c}$ for select cases considered in Figure 4. Figure 5a shows

361 the quasi-static faradaic regime. Here, at low $G z_{c}\left(G z_{c}=0.001\right)$ there is no advective effect, and the upstream and downstream distribution of the overpotential is symmetric. The overpotential in this case extends uniformly across the channel because $v^{2}$ is small, thereby making the lateral distribution controlled by the charge transfer resistance. This figure is representative of the slurry electrode acting purely as a flow battery and not as an EFC. Figure 5b shows a higher $G z_{c}$ where advection is now able to transport the overpotential downstream. Decay in the overpotential as the flow proceeds downstream is apparent though as the faradaic component continues to act as a sink as the slurry proceeds downstream. This is a form of electroactive zone extension (Smith, et al, 2014) that will be discussed more fully in the next subsection. Figure $5 c$ shows a still higher $G z_{c}$ where advection is able to transport the overpotential more and more readily downstream. The decay of the overpotential is less pronounced as the ratio of the faradaic terms to the capacitive advection term (i.e. the capacitive Damköhler number) is now smaller. Finally, Figure 5d shows the spatial distribution of the overpotential for the situation when advection is fully dominant. At this large capacitive Graetz number $\left(G z_{c}=10\right)$, no discernable faradaic-related features exist and the slurry electrode begins to act more and more like an EFC.

(a)

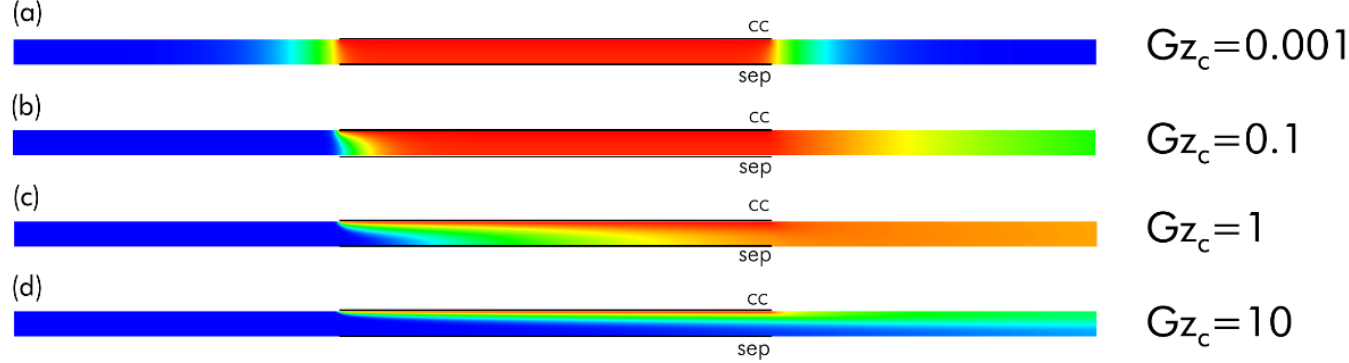

0.0

$\eta^{\prime}$ (nondimensional overpotential)

Figure 5. Contour plots of nondimensional overpotential at selected capacitive Graetz numbers for the same conditions as in Figure $4\left(\gamma=100, v^{2}=0.087\right.$, with $\left.\kappa>\sigma\right)$ 
The numerically predicted nondimensional steady-state current versus $G z_{c}$ for another specific set of cases (with $\gamma$ $=100$ and $v^{2}=4.37$ ) is shown in Figure 6 along with the composite scaling as developed above. For this case, $I_{f}{ }^{\prime}>$ $c_{0}^{3 / 2}$. Thus, the scaling behavior from only three regimes is expressed (as in Figure 3). At no point is the $G z_{c}$ scaling of the fully utilized regime ever present. As can be seen though, the numerical solution again closely follows the asymptotic solution, displaying the scaling behavior of each of the three regimes.

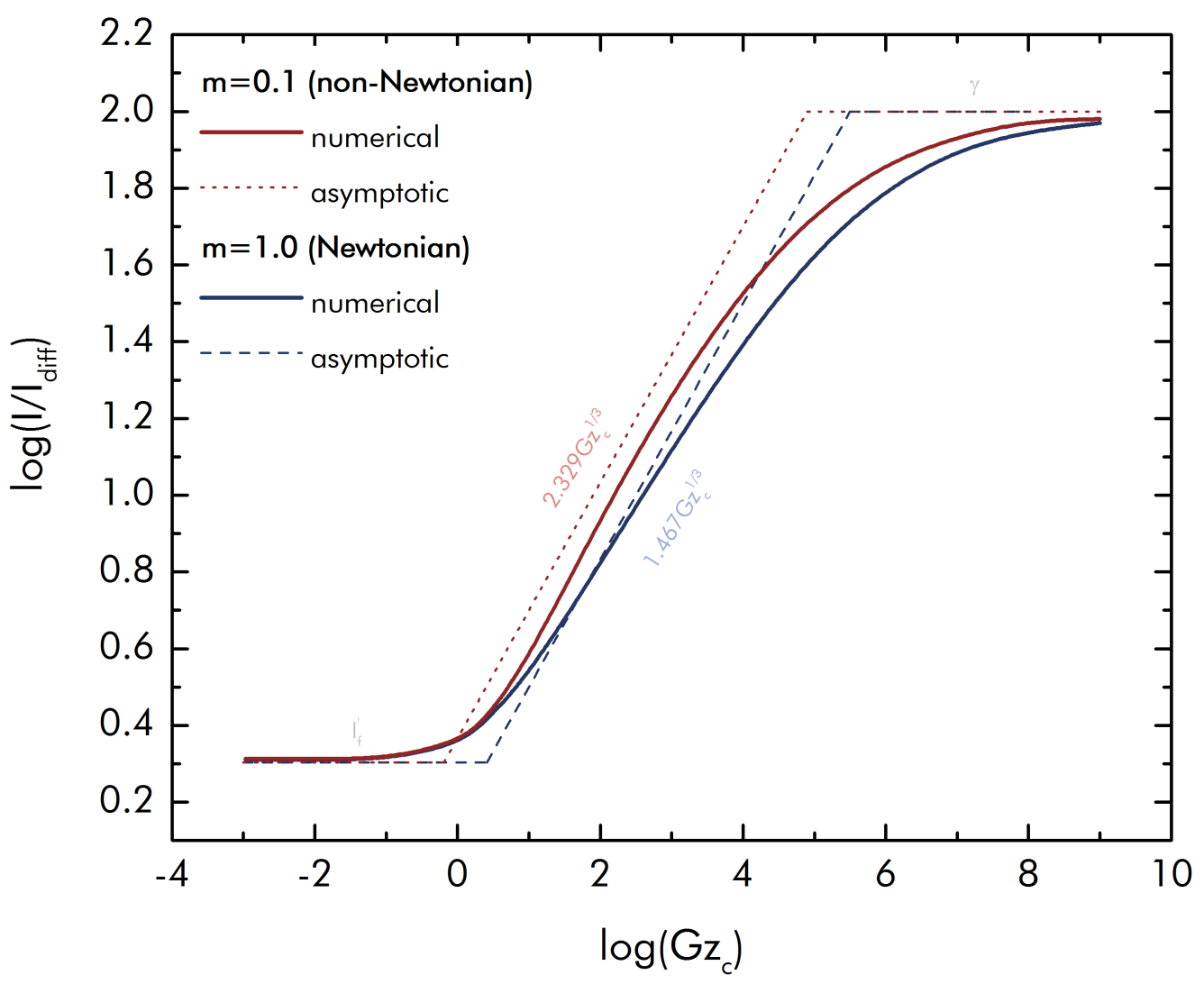

Figure 6. Nondimensional steady-state current $I / I_{\text {diff }}$ versus capacitive Graetz number $\left(G z_{c}\right)$ for $\gamma=100, v^{2}=4.37$. Solid curves represent computational results, while the dashed segments represent the composite scaling solution. As $I_{f}{ }^{\prime}>c_{0}^{3 / 2}$, only three operational regimes are present.

Figure 7 shows the contours of overpotential at various $G z_{c}$ for these conditions $\left(v^{2}=4.37, \gamma=100\right)$ to contrast with the earlier cases in Figure 5 (where $v^{2}<<1$, but $\gamma$ was the same). At very low $G z_{c}$, as in Figure 5 a, the slurry electrode again acts quasi-statically akin to the behavior of a porous electrode. The overpotential does not extend across the entire depth of the channel though because $v^{2}$ is now large enough such that the lateral distribution is no longer controlled exclusively by the charge transfer resistance (i.e. IR-losses are now also significant). Figure 7b shows the contours when $G z_{c}$ is increased to the same value as is Figure 5b. Now, however, the overpotential does not extend 
as far downstream though as the stronger faradaic terms ensure that it decays quickly as the slurry moves away from the electrode. In Figure 7c, the capacitive Graetz number is now large enough that some of advective distortion of the spatial distribution does occur, but the reaction zone still does not progress very far downstream as the charge in the double layer is quickly consumed faradaically. Finally though, as $G z_{c}$ is made larger $\left(G z_{c}=10\right)$, the characteristic overpotential boundary layer behavior of an EFC is expressed, as shown in Figure 7d.

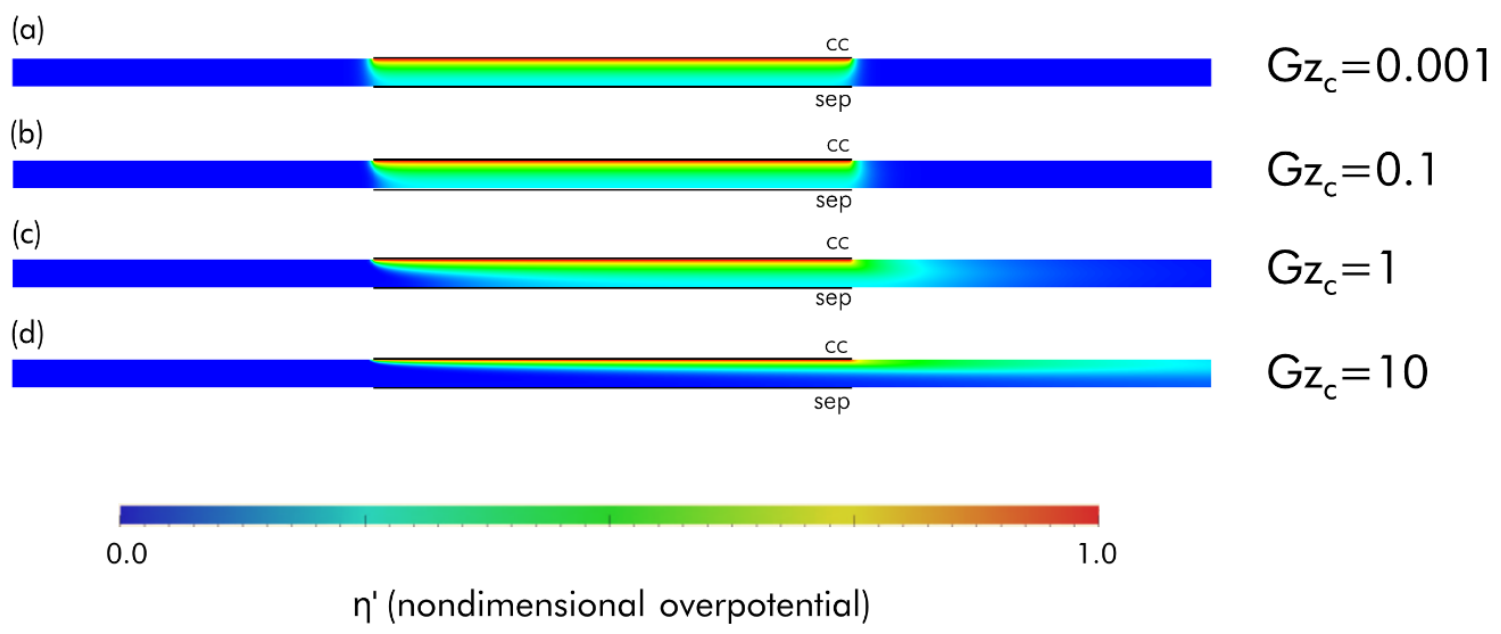

Figure 7. Contour plots of nondimensional overpotential at selected capacitive Graetz numbers for the same conditions as in Figure $6\left(\gamma=100, v^{2}=4.37\right.$, with $\left.\kappa>\sigma\right)$

\subsection{Continuous Flow Electroactive Zone Extension}

Figures 5 and 7 both show how the reaction zone is able to extend well outside of the physical extent of the current collectors - at large enough capacitive Graetz numbers the surface charge is transported well downstream where the associated overpotential continues to cause the faradaic reactions to persist. This phenomena, wherein reactions occur away from the current collectors, was termed electroactive zone extension and was studied for stationary slurries by Smith, et al (2014b). The phenomenon exists because the percolative electronic network of the slurry extends far up and downstream allowing faradaic reactions to persist in regions outside of the extent of the current

410 collectors. These extended regions were termed side zones. In the work of Smith, et al. the existence of electroactive

411 zone extension was found to affect the energy efficiency of a flow battery through mechanisms such as Joule heating 
412

413

414

415

416

417

$\frac{\partial \eta^{\prime}}{\partial x^{\prime}}=\frac{1}{P e_{c}} \frac{\partial^{2} \eta^{\prime}}{\partial x^{2}}-\frac{v^{2}}{P e_{c}} \eta^{\prime}$

The analytical solution for this equation in the side zones shows that the profile of the overpotential along the $x$ -

$421 \quad \eta^{\prime} \sim \exp \left[\frac{x^{\prime}}{2}\left(P e_{c} \pm \sqrt{P e_{c}^{2}+4 v^{2}}\right)\right]$

from the additional Ohmic losses required to utilize the side zones. The nature of the extended electroactive zone, though, is altered for a continuously flowing slurry electrode.

The size of the extended side zones for a flowing slurry electrode with significant overpotential advection can be examined using Equation 17. For the regions upstream and downstream of the current collector, a one-dimensional equation (in the longitudinal direction) describing the evolution of the steady-state overpotential can be formed as direction is described by either exponential growth (upstream of the current collector) or exponential decay (downstream of the current collector) of the form

Depending on the magnitude of the quantity $v^{2} / P e_{c}{ }^{2}$, the length scales of the exponentially growing or decaying side zones take on the values shown in Table 1 . For $v^{2} / P e_{c}^{2}>>1$ (i.e. a situation where the charge transfer resistance is relatively small), both the upstream and downstream side zones have a nondimensional length scale of $1 / v$. The upstream and downstream side zone sizes in this instance are identical and not functions of $P e_{c}$ because the directional transport from advection is negligible. This dependence on $v$ explains why, compared to Figure 4, there is a closer agreement in Figure 6 between the one-dimensional prediction for the quasi-static current $I_{f}$ ' and the numerical prediction at low $G z_{c}$. With smaller $v$, the quasi-static electroactive zone for the $v^{2}=0.087$ case is able to extend farther away from the electrode (as can be seen by comparing the extent of the active zones outside of the leading and trailing edge of the current collector in Figures 4a and 6a), thereby permitting a greater current than what is predicted from the one-dimensional approach.

On the other hand, for $v^{2} / P e_{c}{ }^{2}<<1$, the upstream nondimensional length scale is $1 / P e_{c}$ while the downstream length scale is $1 / D a_{c}$ (i.e. the inverse of the capacitive Damköhler number). Now with participatory advection effects, the upstream and downstream side zone sizes are clearly different. As the relative magnitude of advection is increased, the size of the downstream side zone gets larger and larger (in proportion to $P e_{c}$ ), but conversely, the upstream side 
zone gets smaller and smaller (inversely proportional to $P e_{c}$ ) as upwind diffusion becomes more and more difficult. The complete absence of faradaic reactions results in an infinite downstream length scale; for these cases, corresponding to ideal EFCs, there are no reaction species to consume the double layer charge and there is no associated decay of the overpotential.

Table 1. Nondimensional length scale associated with extended electroactive side zones of a slurry electrode with combined faradaic and advectively-induced capacitive currents

\begin{tabular}{|c|c|c|}
\cline { 2 - 3 } \multicolumn{1}{c|}{} & downstream & upstream \\
\hline$v^{2} / P e_{c}{ }^{2}>>1$ & $1 / v$ & $1 / v$ \\
\hline$v^{2} / P e_{c}{ }^{2}<<1$ & $1 / D a_{c}$ & $1 / P e_{c}$ \\
\hline
\end{tabular}

For cases with conditions corresponding to Figures 4 and $5\left(\gamma=100, v^{2}=0.087\right)$, the analytical solution for the growth and decay of the overpotential upstream and downstream of the electrode (Equation 28) and the numerical profile of the overpotential along the channel midline are plotted in Figure 8 for various values of the capacitive Péclet number. The downstream analytical curve corresponds to the decaying exponential term from Equation 29 with the boundary condition $\eta^{\prime}=1$ at the trailing edge of the current collector. The upstream analytical curve corresponds to the growing exponential term from Equation 28 with the boundary condition $\eta^{\prime}=1$ at the leading edge of the current collector. The numerical and analytical curves show excellent agreement, and the effect of the magnitude of advection is clearly discernable. The decay of the overpotential downstream occurs over increasingly longer distances as the magnitude of advection is increased, while the growth of the overpotential immediately upstream of the leading edge of the current collector clearly requires less and less distance. 


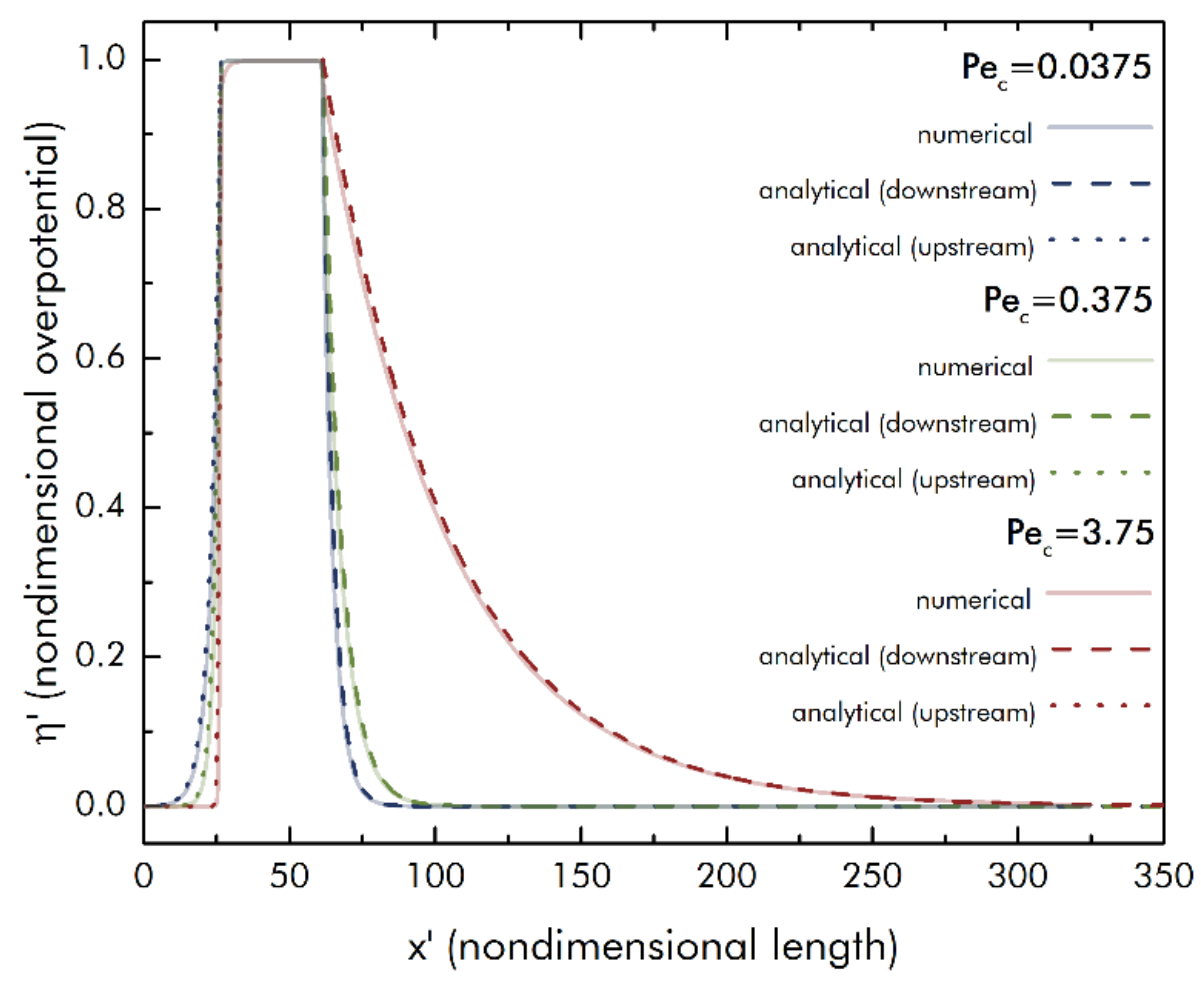

454

455

456

457

458

459

460

461

Figure 8. Analytical and numerically calculated growth and decay of the overpotential upstream and downstream of the current collector. Extent of current collector is from $x^{\prime}=26.3$ to $x^{\prime}=61.4$. As the magnitude of convection is increased, the overpotential extends farther and farther downstream, but its upstream extent is made increasingly smaller.

The characteristics of electroactive zone extension in flowing systems are thus different from stationary systems. In the stationary systems of Smith, et al. (2014b), the existence of the extended electroactive zones was found to cause reduced energy efficiency as the consumption of reactant within the bounds of the current collector forced the reaction zone to extend farther and farther away from the electrode, thereby increasing the associated Ohmic losses.

For flowing slurry electrode systems, however, the fact that the electroactive zone is able to extend upstream and downstream actually results in smaller losses. In cases with large $v^{2} / P e_{c}{ }^{2}$, the extended side zones simply act as additional routes through which the transfer of charge can take place; the IR-losses associated with charge propagation through the side zones may be larger than those resulting from a more direct route across the channel, but the lower local current densities in these extended regions make the net drop in overpotential the same. The fact that the size of the side zone is proportional to $1 / v$ confirms this: for situations with relatively large charge transfer resistance compared to Ohmic losses (i.e. small $v^{2}$ ), the side zone becomes very large as the charge is willing to travel greater and greater distances to find available area through which to react. Conversely, for situations with 

demands a very direct path across the channel.

When $v^{2} / P e_{c}{ }^{2}$ is small, the existence of the side zones improves cell performance even further. In this situation, the nonfaradaic capacitive current (i.e. advection of the overpotential) allows for the reaction zone to extend very long distances downstream where the reactions can continue to take place (perhaps even into the tanks where the reactants are stored). The increased interfacial area made available for reactions within the side zone thus offers tremendous advantages in terms of the reduction of kinetic losses. Furthermore, as the counterion associated with the nonfaradaic capacitive current has already completed its path across the separator (having done so entirely within a region near to the current collector), there is no additional Ohmic loss associated with the fact that the faradaic reactions continue to occur so far downstream — the necessary charge has instead been transferred downstream by the advective mechanism. Figure 9 shows schematic diagram of this process. Downstream the overpotential associated with the double layer is simply consumed by the faradaic reaction current and relaxes back to the open circuit potential — there is no requirement for charge to be ohmically transferred large distances up and downstream.

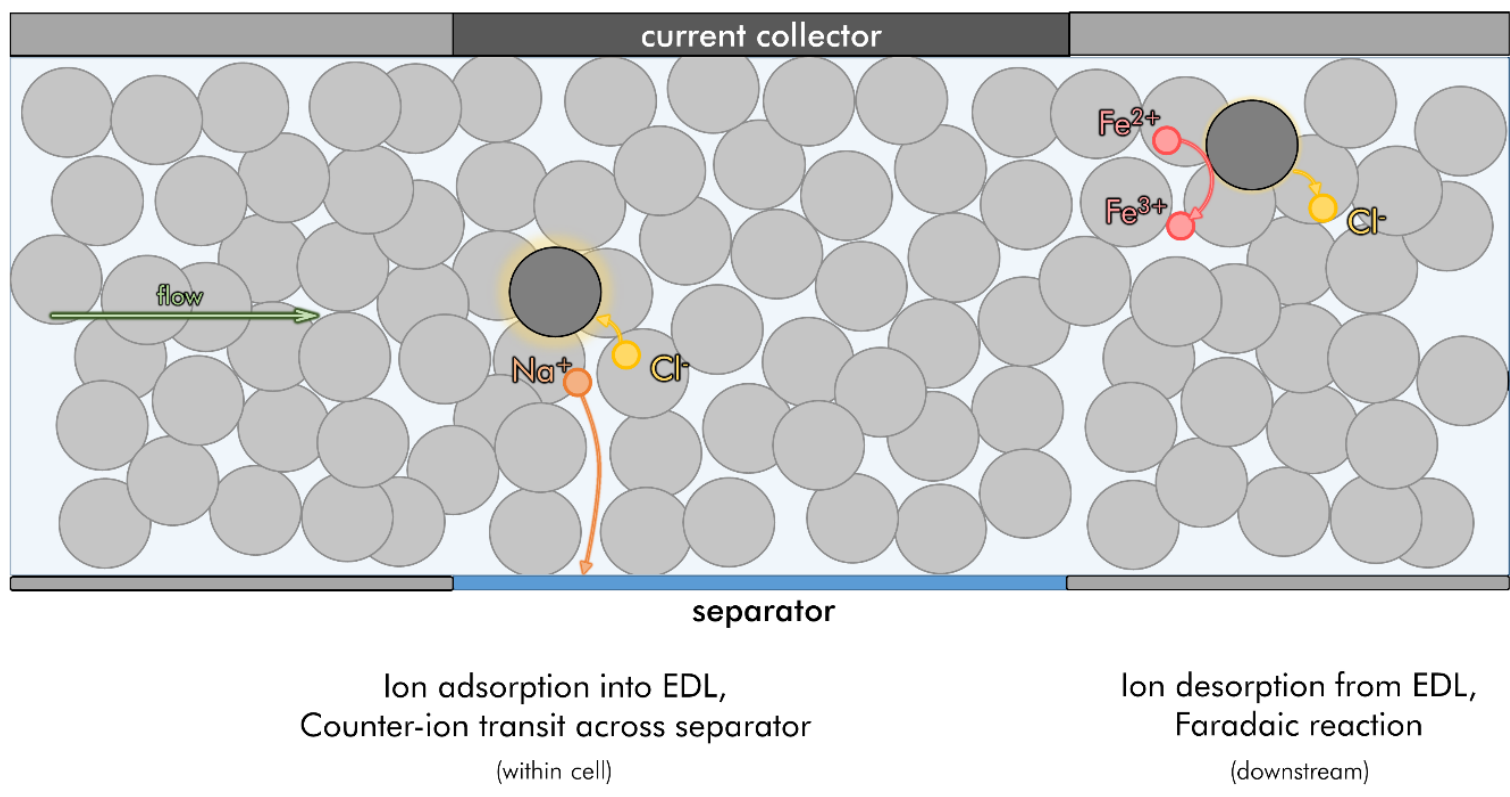

Figure 9. Diagram showing the downstream relaxation of the overpotential back to the open circuit potential. For demonstration purposes, the ions associated with the ferric/ferrous chloride redox couple with sodium chloride supporting electrolyte have been used. 
In this paper, a set of equations describing flowable slurry electrodes taking into account both faradaic reactions and

491 surface charge advection was delimited. These equations have applicability to electrochemical devices using

492 flowable slurry electrodes, including flow batteries, electrochemical flow capacitors, and FCDI systems.

493 Simplifications were made to these equations that allowed the electrochemical behavior of a slurry electrode to be

494 described by the interaction of three principal terms: a Laplacian term describing the diffusion of overpotential, a

495 total derivative term describing the advection of overpotential, and a volumetric sink/source describing the faradaic

496 consumption of the overpotential. These equations were nondimensionalized and the steady-state current able to be

497 supported by a flowing slurry electrode was shown to be dependent only on the capacitive Graetz number, the

498 nondimensional exchange current density, a ratio of the ionic and electronic conductivities, and the flow behavior

499 index. Depending on the respective values of these nondimensional parameters, an electrochemical flow cell with

500 slurry electrodes was shown to operate in one of at most four regimes: a quasi-static regime, a capacitively fully

501 utilized regime, an overpotential boundary layer development regime, or an Ohmically-limited regime. Asymptotic

502 scaling solutions to predict the steady state current were developed for each of these regimes, and good agreement

503 with numerical simulations was found. In turn, the newly-introduced capacitive Damköhler number was found to be

504 important in describing whether an electrochemical cell with slurry electrodes acts more like a flow battery or like

505 an electrochemical flow capacitor

506 Finally, the phenomenon of electroactive zone extension in a slurry electrode was examined for the regions

507 immediately up and downstream of the current collector. For cases with negligible advection, the size of both the

508 upstream and downstream side zones was found to be identical and related only to the nondimensional exchange

509 current density. As the relative magnitude of advection increases though, it was found that the side zones become

510 asymmetric and dependent on the value of both the nondimensional exchange current density and the capacitive

511 Péclet number, as expressed by the capacitive Damköhler number. 
512 The models and scaling relationships that were developed in this paper to predict the achievable current density from

513 electrochemical cells containing slurry electrodes can aid in optimal particle selection and in the design of flow cells

514 tailored to specific EFC, flow battery, or FCDI applications.

515

\section{Acknowledgements}

517 This work was performed under ARPA-E contract DE-AR0000352.

518

\section{$519 \quad$ List of Symbols}

$520 \quad a_{0} \quad$ specific interfacial areal density $\left(\mathrm{m}^{2} / \mathrm{m}^{3}\right)$

$521 \quad A R \quad$ aspect ratio of the channel $(=L / \delta)$

$522 \quad c \quad$ electrolyte concentration

$523 \quad c_{0} \quad$ constant $(=(6(2+1 / m) / 2 \Gamma(4 / 3))$

$524 \quad C_{d l} \quad$ capacitance per unit interfacial area $\left(\mathrm{F} \mathrm{m}^{-2}\right)$

$525 D a_{c} \quad$ capacitive Damköhler number: [overpotential source/sink]/[overpotential advection]

$526 \quad D_{e} \quad$ diffusion coefficient of electrolyte $\left(\mathrm{m}^{2} \mathrm{~s}^{-1}\right)$

$527 D_{\text {eff }} \quad$ effective diffusion coefficient of the electrolyte $\left(\mathrm{m}^{2} \mathrm{~s}^{-1}\right)$

$528 E_{\text {ref }} \quad$ open-circuit potential of electrode reaction

$529 \quad F \quad$ Faraday's constant

$530 \quad G z_{c} \quad$ capacitive Graetz number ( = Pe $\left./ A R\right)$

$531 \quad i_{0} \quad$ exchange current density $\left(\mathrm{A} \mathrm{m}^{-2}\right)$

$532 \quad i_{1} \quad$ current density in the particle phase

$533 \quad i_{2} \quad$ current density in the solution phase

$534 I_{\text {diff }}$ diffusional current scale

$535 \quad I_{f}{ }^{\prime} \quad$ nondimensional current

$536 j \quad$ volumetric reaction current $\left(\mathrm{A} \mathrm{m}^{-3}\right)$

$537 \quad K \quad$ flow consistency index $\left(\mathrm{Pa} \mathrm{s}^{\mathrm{n}}\right)$ 


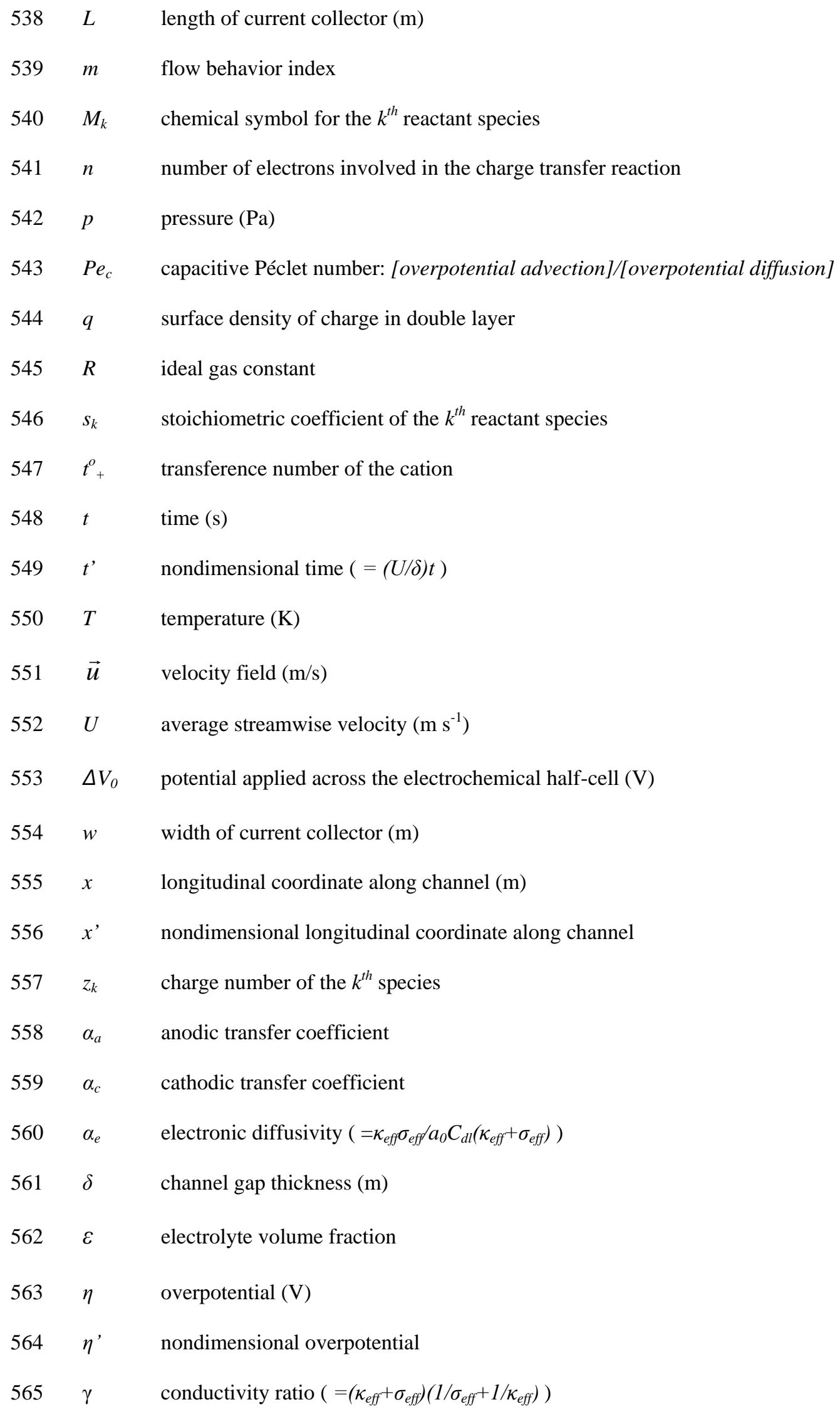




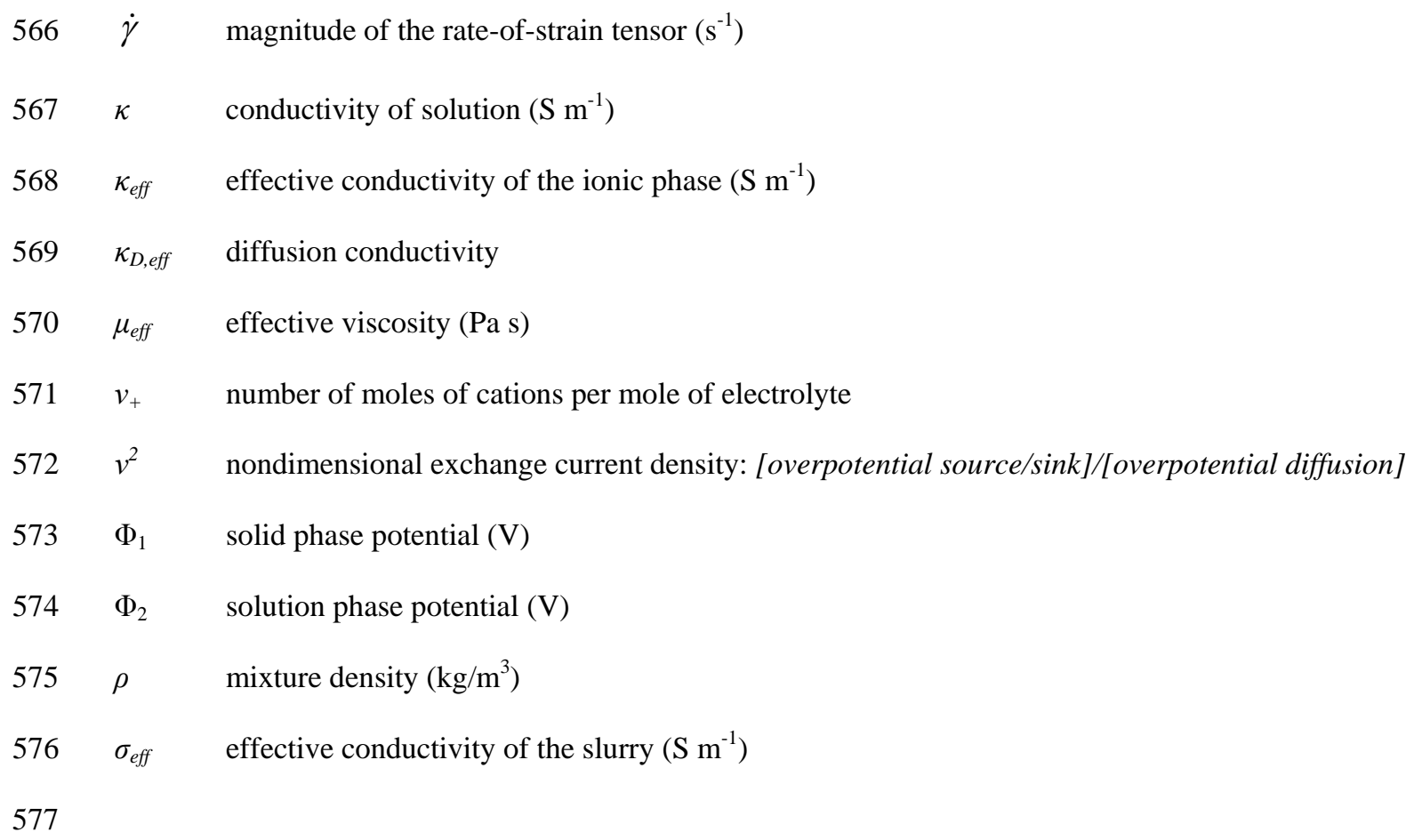




\section{References}

Appleby, A., \& Jacquier, M. (1976). The C.G.E. circulating zinc/air battery: A practical vehicle power source. Journal of Power Sources, 1(1), 17-34.

Baria, D., \& Hulburt, H. (1973). Reduction of Crotonic Acid with Hydrogen on a Slurry Electrode. J. Electrochem. Soc., 120(10), 1333-1339.

Biesheuvel, P. M., Fu, Y., \& Bazant, M. Z. (2012). Electrochemistry and Capacitive Charging of Porous Electrodes in Asymmetric Multicomponent Electrolytes. Russian Journal of Electrochemistry, 48(6), 645-658.

Biesheuvel, P., Fu, Y., \& Bazant, M. (2011). Diffuse charge and Faradaic reactions in porous electrodes. Physical Review E, 83(6).

Brunini, V., Chiang, Y., \& Carter, W. (2012). Modeling the hydrodynamic and electrochemical efficiency of semisolid flow batteries. Electrochimica Acta, 69, 301-307.

Dennison, C., Gogotsi, Y., \& Kumbur, E. (2014). In situ distributed diagnostics of flowable electrode systems: resolving spatial and temporal limitations. Phys. Chem. Chem. Phys., 16(34), 18241-18252.

Devan, S., Subramanian, V., \& White, R. (2004). Analytical Solution for the Impedance of a Porous Electrode. J. Electrochem. Soc., 151(6), A905-A913.

Duduta, M., Ho, B., Wood, V., Limthongkul, P., Brunini, V., Carter, W., \& Chiang, Y. (2011). Semi-Solid Lithium Rechargeable Flow Battery. Adv. Energy Mater., 1, 511-516.

Fan, F., Woodford, W., Li, Z., Baram, N., Smith, K., Helal, A., . . Chiang, Y. (2014). Polysulfide Flow Batteries Enabled by Percolating Nanoscale Conductor Networks. Nano Letters, 14, 2210-2218.

Fleischmann, M., \& Oldfield, J. (1971). Fluidized Bed Electrodes, Part I: Polarization Predicted by Simplified Models. J. Electroanal. Chem., 29, 211-230.

Foller, P. (1986). Improved slurry zinc/air systems as batteries for urban vehicle propulsion. Journal of Applied Electrochemistry, 16(4), 527-543.

Gabrielli, C., Huet, F., Sahar, A., \& Valentin, G. (1994). Dynamic analysis of charge transport in fluidized bed electrodes: impedance techniques for electroactive beds. Journal of Applied Electrochemistry, 24, 481-488.

Gu, W., Wang, C., Weidner, J., Jungst, R., \& Nagasubramanian, G. (2000). Computational Fluid Dynamics Modeling of a Lithium/Thionyl Chloride Battery with Electrolyte Flow. J. Electrochem. Soc., 147(2), 427434.

Hatzell, K., Boota, M., Kumbur, E., \& Gogotsi, Y. (2015). Flowable Conducting Particle Networks in Redox-Active Electrolytes for Grid Energy Storage. J. Electrochem. Soc., 162(5), A5007-A5012.

Heydecke, G., \& Beck, F. (1990). A Parametric Study of the Carbon Black Slurry Electrode. Carbon, 28(2/3), 301309.

Hoyt, N., Wainright, J., \& Savinell, R. (2015a). Mathematical Modeling of Electrochemical Flow Capacitors. J. Electrochem. Soc., 162(4), A652-A657.

Hoyt, N., Wainright, J., \& Savinell, R. (2015b). Current Density Scaling in Electrochemical Flow Capacitors. J. Electrochem. Soc. 
614

615

616

617

618

619

620

621

622

623

624

625

627

628

629

630

631

632

633

634

635

636

637

638

Jeon, S., Park, H., Yeo, J., Yang, S., Cho, C., Han, M., \& Kim, D. (2013). Desalination via a new membrane capacitive deionization process utilizing flow-electrodes. Energy \& Environmental Science, 6(5), 14711475.

Johnson, A., \& Newman, J. (1971). Desalting by Means of Porous Carbon Electrodes. J. Electrochem. Soc., 118(3), 510-517.

Macosko, C. (1994). Rheology: Principles, Measurements, and Applications. Hoboken, NJ: Wiley.

Newman, J. (1991). Electrochemical Systems 2nd Edition. New Jersey: Prentice Hall.

Newman, J., \& Tiedemann, W. (1975). Porous-Electrode Theory with Battery Applications. AIChE Journal, 21(1), 25-41.

Newman, J., \& Tobias, C. (1962). Theoretical Analysis of Current Distribution in Porous Electrodes. J. Electrochem. Soc., 109(12), 1183-1191.

OpenFOAM. (2012). OpenFOAM User Guide v2.0.1. available from http://foam.sourceforge.net/docs/Guidesa4/UserGuide.pdf.

Petek, T., Hoyt, N., Wainright, J., \& Savinell, R. (2015). Slurry Electrodes for Iron Plating in an All-Iron Flow Battery. J. Power Sources, 294, 620-626.

Petek, T., Hoyt, N., Wainright, J., \& Savinell, R. (2016). Characterizing Slurry Electrodes Using Electrochemical Impedance Spectroscopy. J. Electrochem. Soc., 163(1), A5001-A5009.

Presser, V., Dennison, C., Campos, J., Knehr, K., Kumbur, E., \& Gogotsi, Y. (2012). The Electrochemical Flow Capacitor: A New Concept for Rapid Energy Storage and Recovery. Advanced Energy Materials, 2(7), 895-902.

Richardson, S. (1979). Extended Leveque Solutions for Flows of Power Law Fluids in Pipes and Channels. Int. J. Heat Mass Transfer, 22, 1417-1423.

Smith, K., Brunini, V., Dong, Y., Chiang, Y., \& Carter, W. (2014b). Electroactive-Zone Extension in Flow-Battery Stacks. Electochim. Acta, 147, 460-469.

Smith, K., Chiang, Y., \& Carter, W. (2014a). Maximizing Energetic Efficiency in Flow Batteries Utilizing NonNewtonian Fluids. J. Electrochem. Soc., 161(4), A418-A496.

Trainham, J., \& Newman, J. (1977). A Flow-through Porous Electrode Model: Application to Metal-Ion Removal from Dilute Streams. J. Electrochem. Soc., 124(10), 1528-1540.

Wang, C., Gu, W., \& Liaw, B. (1998). Micro-Macroscopic Coupled Modeling of Batteries and Fuel Cells. J. Electrochem. Soc., 145(10), 3407-3416.

Wu, S., Yongfu, Z., Li, D., Xia, Y., \& Si, S. (2015). An asymmetric Zn//Ag doped polyaniline microparticle suspension flow battery with high discharge capacity. Journal of Power Sources, 275, 305-311. 